\title{
Vibration-induced muscle fatigue, a possible contribution to musculoskeletal injury
}

Accepted: 23 May 2002 / Published online: 27 August 2002

(C) Springer-Verlag 2002

\begin{abstract}
Localized muscle fatigue resulting from 30-min sustained and intermittent grip exertions of 5\% maximal voluntary contraction (MVC) with and without hand-vibration exposure $(10 \mathrm{~Hz}, 7 \mathrm{~mm}$ displacement amplitude) was investigated. Muscle fatigue was quantified by the magnitude of the twitch force elicited in the right flexor digitorum superficialis muscle of the long finger using the low-frequency fatigue (LFF) method. The influence of vibration in the sustained grip exertion condition exacerbates fatigue as seen with the reduction in twitch force 30-60 min post-work task. Intermittent low grip force exertion conditions with and without vibration exposure show negligible fatigue, suggesting the benefit of rest in the work cycle. Perception of muscle fatigue was dissociated from the objective measure of twitch force, suggesting that LFF was not perceived. The presence of LFF and the lack of perception of LFF may increase the risk for the development of musculoskeletal disorders. The findings of this study may apply to the design of the work cycles and tasks that require the use of vibratory tools.
\end{abstract}

Keywords Low frequency fatigue $\cdot$ Hand-arm vibration · Musculoskeletal disorders ·

Occupational therapy

D.E. Adamo

Wayne State University, Eugene Applebaum College of Pharmacy and Health Sciences, Occupational Therapy,

139 Shapero Hall, Detroit, MI 48202, USA

B.J. Martin $(\square)$

Department of Industrial and Operations Engineering, Center for Ergonomics, The University of Michigan,

1205 Beal Avenue, Ann Arbor, MI 48109-2117, USA

E-mail: martinbj@umich.edu

Fax: + 1-734-7643451

P.W. Johnson

Department of Environmental Health,

University of Washington, Box 357234, Seattle, WA 98195, USA

\section{Introduction}

Hand-transmitted vibration is associated with several health risks such as vascular, neurological and musculoskeletal disorders (MSDs) (Pelmear 1982; Bovenzi et al. 1987; Engstrom et al. 1990). In addition, vibration-induced changes in proprioceptive and exteroceptive input to peripheral and central sensorimotor loops contribute to alterations in sensory perception and motor performance (Goodwin et al. 1972; Roll et al.1986; Park et al. 1993). Thus far, there have been few attempts to investigate the health effects associated with the sensorimotor response induced by vibration exposure (Martin and Armstrong 1995). We need to consider the potential risks to health associated with the persistence of localized muscle fatigue. Several studies support the theory that fatigue and a lack of fatigue recovery are potential precursors for injury (Edwards et al. 1977; Vøllestad and Sejersted 1988; Martin and Armstrong 1995). Muscle fatigue may result from the influence of vibration exposure as the motor response to the altered sensory messages includes increases in force exertion and/or co-contractions (Martin and Park 1997).

The effects of vibration on the neurological network occur through stimulation of sensory receptors within the cutaneous, muscular and articular structures (Johansson 1978; Roll et al. 1986). The vibration-induced activity of these receptors is considered a leading cause of specific perceptive and sensorimotor effects, such as an alteration in force control (Gauthier et al. 1981) and position and velocity control of visually guided hand movements (Gauthier et al. 1981; Martin et al. 1991; Gerard and Martin 1999).

During gripping tasks, the contribution of the tonic vibration reflex (TVR) (Hagbarth and Eklund 1966) superimposed on ongoing voluntary contractions increases the magnitude of the sustained contraction and subsequent load on the muscles. It has been shown that the contribution of the TVR to the grip exertion 
increases for frequencies up to $100-150 \mathrm{~Hz}$ and decreases beyond this frequency range (Park and Martin 1993). The TVR is also responsible for an alteration in force control, force variability, and force sensation (Gauthier et al. 1981; Jones and Hunter 1985). These phenomena are likely to contribute to muscle stress and fatigue. It is widely accepted that fatigue is task-dependent and is caused by a combination of processes (Edwards et al. 1977; Bigland-Ritchie et al. 1979; Sandercock et al. 1985; Enoka and Stuart 1992; Moussavi et al. 1992; Johnson et al. 1995). Fatigue generated by low force exertions over time (sustained or intermittent $<10 \%$ ) has been primarily associated with a failure in the excitation-contraction coupling mechanism (Sandercock et al. 1985; Enoka and Stuart 1992). This type of fatigue may be quantified by low-frequency stimulation of the muscles (Edwards et al. 1977; Bystrom and Kilbom 1991; Johnson et al. 1995) and is named low-frequency fatigue (LFF). The persistence of LFF may be a precursor for MSDs (Bongiovanni and Hagbarth 1990; Hagberg et al. 1995) since the recovery process may exceed $24 \mathrm{~h}$ (Edwards et al. 1977; Mellor and Stokes 1992; Johnson et al. 1995).

Besides the force exposures, alterations in perception of sensorimotor performance resulting from vibration exposure are not coherently perceived. A sensation of improved performance is often reported (Martin et al. 1980, 1991; Gauthier et al. 1981). The dissociation between perception and performance may further increase the risk for occupational injuries and compromise health.

A large proportion of industrial tasks include the use of hand-controlled vibrating tools, which are associated with MSDs, and many of these tasks may not require excessive grip exertion (Armstrong et al. 2002). Furthermore, the influence of vibration on muscle fatigue is largely unknown. Hence, the objectives of this study were to quantify the influence of hand vibration on muscle fatigue resulting from intermittent and sustained low grip force exertions and determine the relationship between LFF and fatigue perception. It was hypothesized that (1) vibration exacerbates fatigue; (2) fatigue is reduced in intermittent grip exertions, and (3) LFF is not perceived. To test these hypotheses, the objective and subjective measures of muscle fatigue elicited in the finger flexor muscles by intermittent and sustained grip exertions were compared in vibratory and non-vibratory conditions.

\section{Methods}

Vibration frequency and magnitude, grip exertion level and grip contraction patterns (sustained and intermittent) were selected to simulate common work tasks involving the use of vibrating tools. The values selected in the present experiment were derived from measures collected in real work environments. In this context, the LFF method was used to quantify the effects of low grip force exertion (Edwards et al. 1977; Bystrom and Kilbom 1991; Johnson 1998).

\section{Participants}

Twenty subjects (11 male, 9 female) free from any neurological disorders and with no history of hand-arm vibration syndrome participated in this experiment as paid volunteers. The subjects were non-smoking, free of cardiovascular medication, and between 20 and 40 years of age. All participants signed an informed consent agreement approved by the ethics committee of the University of Michigan prior to their inclusion in the study.

\section{Twitch force recording}

The subjects were seated in a comfortable armchair in an upright posture, body weight was equally distributed and feet were flat on a supporting surface. The right arm was resting on an adjustable table supporting the twitch force measuring apparatus (Fig. 1). The elbow angle was set in the $120-140^{\circ}$ range to obtain full forearm supination. A metal plate was placed over the hypothenar eminence to stabilize the supinated forearm posture in relaxed position, and thus maintenance of the supinated position did not require any voluntary effort from the subject. Twitch force in the compartment of the flexor digitorum superficialis (FDS) muscle corresponding to the right long finger was obtained in response to submaximal electrical stimulation of the muscle and recorded by a load cell mounted over the long finger. The load cell was attached to a fixed frame through an adjustable rod, allowing it to be positioned to accommodate anthropometric variations between subjects, and placed perpendicular to the palmar surface at the midpoint between the distal and proximal interphalangeal (DIP and PIP) joints of the long finger. The adjacent digits were stabilized under Velcro strapping (Fig. 1).

The general location of the $7.5 \mathrm{~mm}$ stimulation electrode (In Vivo Metric) was first determined by performing palpation and resistive maneuvers on the subject to isolate the FDS muscle. This was followed by systematic displacement of the stimulation electrode along a grid pattern in the region of the muscle. The optimal location of the stimulation electrode was determined by the maximum twitch force generated in the FDS muscle that occurred simultaneously with minimal variation of twitch magnitude, relative to the placement of the electrode. In addition, the final placement of the stimulation electrode was determined by the subjects' reports of maximum sustainable discomfort within the highest level of stimulus intensity tolerated. (This was done in order to recruit the largest number of muscle fibers.) This optimal location was clearly marked, thus allowing identical placement of the stimulation electrode for subsequent experiments.

Electrical pulses of $10-20 \mathrm{~mA}$, and $0.1 \mathrm{~ms}$ in duration were delivered at low frequency $(2 \mathrm{~Hz})$ through a Grass medical stimulator (GS880) connected to an isolation unit (SIU5) and a constant current unit (CCU1A). The $18 \mathrm{~mm} \mathrm{Ag} / \mathrm{AgCl}$ pre-gelled ground electrode (Agilent Technologies) was placed over the right lateral epicondyle.

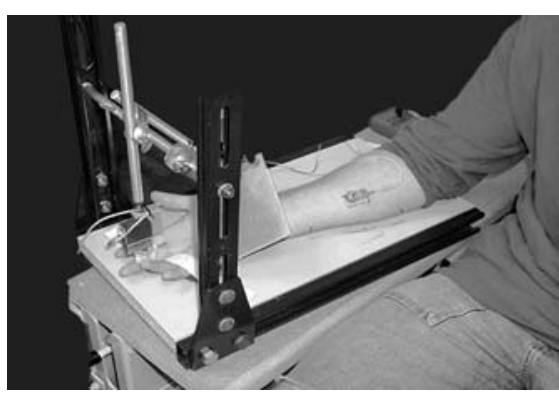

Fig. 1. Low frequency fatigue experimental setup. The load cell placed over the second phalanx of the middle finger measures the twitch force elicited in the flexor digitorum superficialis (FDS). The stimulation electrode was placed over the FDS so as to obtain the largest twitch with minimal variation and discomfort 
Work task

In a seated upright posture the subject grasped a horizontal handle equipped with a strain gauge dynamometer connected to a digital voltmeter. The shoulder was abducted approximately $20^{\circ}$ and the elbow angle was $120^{\circ}$. The forearm was supported and fully pronated, and the wrist was extended approximately $30^{\circ}$ (Fig. 2). The subjects monitored and maintained their grip force exertion by reading the digital display, thus ensuring a constant level over time for all work tasks.

Four experimental conditions were tested: sustained (S) and intermittent (I) grip exertion of $5 \% \mathrm{MVC}$, with (V) and without (NV) vibration exposure. The duration of each work task was 30 min. The intermittent grip exertion condition consisted of a 5-min work, 1-min rest cycle repeated five times. The handle was attached to the vibrator. In the vibration conditions, vibration was applied perpendicularly to the handle, along the axis of the forearm. The vibrator was actuated by a sine wave signal generated through a vibration monitor (Trig-tek $610 \mathrm{M}$ ), signal compressor (Trig-tek 801B) and a power amplifier (Tecron 5530). This servo control system was used to maintain a constant vibration displacement amplitude. The vibration frequency was $10 \mathrm{~Hz}$ and the displacement amplitude $7 \mathrm{~mm}$.

\section{Subjective evaluation of muscle fatigue}

Subjective perception of localized muscle fatigue in the right hand and forearm was rated on a bipolar scale by placing a mark next to the number and corresponding verbal descriptor that most closely matched the level of perception (Fig. 3).

\section{Procedure}

All subjects were instructed to minimize physical exertions $24 \mathrm{~h}$ prior to the experiment. Activities such as lengthy computer use as well as tasks requiring a high level of physical exertion were



Fig. 2. Setup of the work fatiguing task

\begin{tabular}{|l|l|l}
\hline & 1.0 & None \\
\hline & 1.5 & \\
\hline & 2.0 & Marginal \\
\hline & 2.5 & \\
\hline & 3.0 & Slightly \\
\hline & 3.5 & \\
\hline & 4.0 & Somewhat \\
\hline & 4.5 & \\
\hline & 5.0 & Noticeable \\
\hline & 5.5 & \\
\hline & 6.0 & Significant \\
\hline & 6.5 & \\
\hline & 7.0 & Extreme \\
\hline
\end{tabular}

Fig. 3. Bipolar rating scale. Each subject indicated on the scale the perception of muscle fatigue in their right forearm prior to each LFF measure strongly discouraged. On the first experimental day, the subjects were introduced to the experiment, signed the consent forms, and then the MVC was measured in the work task posture prior to the first experimental condition. Two trials of approximately 5-s duration and separated by a 3-min rest period were performed. Each trial consisted of: an initial 1-s rest period, followed by a 1 - to 2 -s force build-up and a 2- to 3-s maximum exertion. The highest grip force level was used as the MVC reference for all experimental conditions.

For each experimental day, the subjective perception of muscle fatigue and the objective measure of twitch force were evaluated before the task began, and then immediately, 30, 60 and $120 \mathrm{~min}$ post-work task, as illustrated by the experimental time line (Fig. 4)

The magnitude of the twitch force (the indicator of muscle fatigue) was calculated as the average of three 30 -impulse trains following a 2-3 min potentiation period during which the twitch force increases monotonically before reaching a stable plateau (Johnson et al. 1995). The subjects were exposed to one of the four randomly assigned 30-min experimental work tasks, which were presented on non-consecutive days. A randomized block design was used to control for any potential order effect.

\section{Data analysis}

Using subjects as their own control, a repeated measures analysis of variance (ANOVA) was performed on the average twitch force measures obtained in each condition and between conditions, to determine the effects of vibration exposure and work cycles on muscle fatigue. A Dunnett's test $(\alpha=0.05)$ was also used to compare the control (pre-work) measure with the post-work task measures. An ANOVA and a Dunnett's test $(\alpha=0.05)$ were also performed on subjective rating measures to determine the influence of the experimental conditions on subjective perception.

\section{Results}

\section{Twitch force}

The sustained grip exertion with vibration condition (SV) showed the greatest decrease of twitch force when compared to all other conditions and this was seen 60 min post-work task. This was followed by the sustained grip exertion without vibration condition (SNV). The intermittent grip exertion with vibration condition (IV) showed some muscle fatigue 30 min post-work task and recovery to pre-work task measures occurred sooner than in the SV and SNV conditions. No fatigue was observed in the intermittent grip exertion condition without vibration (INV). No gender effect was observed in the present study $(F=0.441 ; P=0.520)$; hence data were pooled for measuring the effects of the experimental conditions on fatigue.

The ANOVA, presented in Table 1, indicated a significant influence of the experimental conditions on the

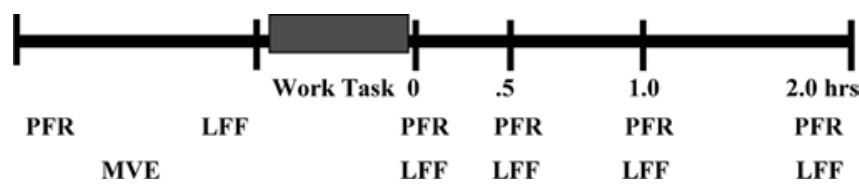

Fig. 4. Experimental time line. Time intervals at which maximum voluntary contraction $(M V C)$, twitch force $(L F F)$ and subjective perception of muscle fatigue rating $(P F R)$ are recorded 
twitch force $(P=0.018)$. Grip exertion conditions were significant $(P=0.007)$ whereas interaction between grip exertion and vibration exposure were not significant $(P=0.475)$. Vibration was not significant $(P=0.325)$ but, as demonstrated when comparing like contraction types, fatigue was greater in the presence of vibration (Fig. 5). Further, statistical analysis compared the prework task twitch force measure with the time interval that indicated the greatest decrease in the magnitude of the twitch force. This corresponded to $30 \mathrm{~min}$ post-work in the IV and INV conditions and 60 min post-work task in the SV and SNV conditions. This result is in agreement with previous studies of LFF (Johnson et al. 1995; Johnson 1998).

The SV condition showed a significant decrease in twitch force of $12.5 \%$ followed by the SNV condition, which induced a modest but non-significant decrease in twitch force of $7.5 \%$ with a relatively full recovery. In the INV condition alone a negligible decrease in twitch force of $4.2 \%$ occurred, while IV exposure induced only a $5 \%$ decrease in twitch force. In both intermittent grip conditions, twitch force measures $2 \mathrm{~h}$ post-work were within $4 \%$ of the pre-work values, suggesting almost full recovery.

\section{Perception of muscle fatigue}

The ANOVA indicated a significance influence of the experimental conditions on perception of muscle fatigue $(P=0$. 001) when compared to the pre-work measurement. The differences were statistically significant in all

Table 1. ANOVA results for twitch force measures $60 \mathrm{~min}$ postwork task

\begin{tabular}{llll}
\hline Condition & $d f$ & $F$ & $\begin{array}{l}\text { Signifi- } \\
\text { cance }\end{array}$ \\
\hline Vibration & 1 & 1.042 & 0.325 \\
Grip & 2 & $5.987^{*}$ & 0.007 \\
Vibration $\times$ Grip & 2 & 0.539 & 0.475 \\
All conditions & 4 & $3.239^{*}$ & 0.018 \\
\hline
\end{tabular}

$* P<0.05$

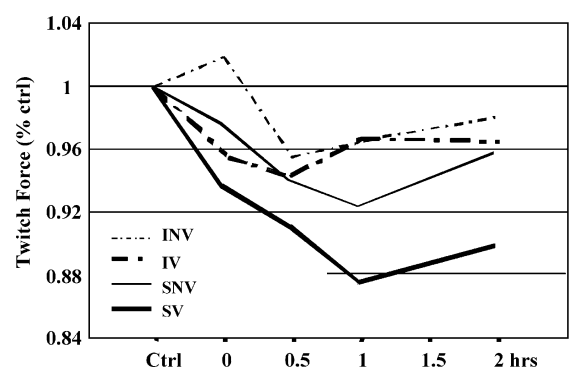

Fig. 5. Average twitch force response of all subjects in all conditions as a function of time. The vibration/ sustained grip exertion $(S V)$ condition elicits the greatest magnitude of twitch force reduction. ( $S$, sustained; $I$, intermittent; $V$, vibration; $N V$, no vibration) experimental conditions $(P<0.001)$. In the SV and SNV conditions there was an approximate increase of 3 points, with an average maximum score of 4.10 (1.8) with vibration and 4.07 (1.5) without vibration, on a 7.5point rating scale immediately post-work task. In both the IV and INV conditions there was an approximate increase of 2 points, with an average maximum score of 3.0. A decrease below 2 points on the rating scale was observed $60 \mathrm{~min}$ post-work task for all conditions, suggesting a recovery of the type of fatigue perceived.

The results of the Dunnett's test showed that perception of muscle fatigue was significantly higher in the SV condition when compared to the IV and INV. The difference between SV and SNV conditions, and the IV and INV conditions did not show any significant effect. These findings are described in the graph in Fig. 6, which illustrates that the greatest differences are between the sustained and intermittent grip exertion conditions.

\section{Discussion}

The major findings of the study showed that hand vibration superimposed on sustained low grip force exertion exacerbates fatigue. Sensorimotor and biomechanical mechanisms are likely to contribute to an increase in muscle contractions and thus to the development of fatigue. The vibration-induced effect is negligible for intermittent grip exertion that allowed for a 1-min rest for every 5 min of work. Furthermore, muscle fatigue was only perceived immediately post-work task in all conditions, indicating a decoupling with the occurrence of LFF at $30-60$ min post-work task. This phenomenon indicates dissociation between perception of muscle fatigue and motor effects.

\section{Motor effects associated with vibration}

The largest decrease in twitch force occurred $1 \mathrm{~h}$ postwork task in the SV condition, when compared to all other time intervals and conditions. Maximum fatigue occurred $1 \mathrm{~h}$ post-work in the continuous work cycles and 30 min post-work in the intermittent work cycles.

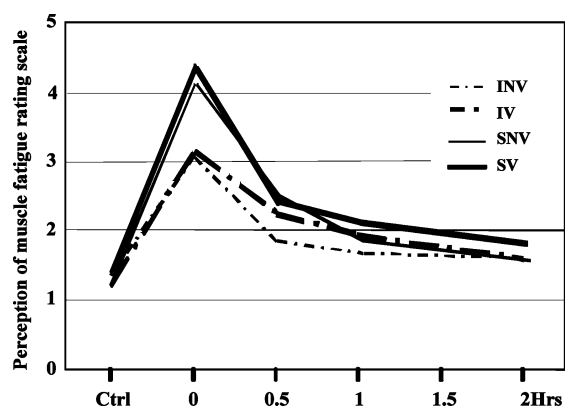

Fig. 6. Perception of fatigue is higher immediately after the work task and then dissipates quickly. ( $S$, sustained; $I$, intermittent; $V$, vibration; $N V$, no vibration) 
Recovery occurred sooner in the intermittent work cycles than in the continuous work cycles. Vibration exposure during sustained exertion induced a larger decrease in the twitch force when compared to the corresponding no-vibration condition; however, statistical significance was not reached. This result can be related to the post-contraction potentiation effect (Vandervoort et al. 1983). Indeed, four subjects exhibited a systematic post-work potentiation in all conditions. This phenomenon, known to co-exist with fatigue (Gardner et al. 1989; Green and Jones 1989), is most likely evidenced by the increase in twitch force observed immediately postwork in the INV condition where fatigue was negligible. Thus, post-contraction potentiation would be maximal in the measurement immediately post-work and would co-exist and possibly limit or compensate for any decrease in twitch force. Hence, despite a marginal statistical result, it can be assumed that vibration can influence and exacerbates muscle fatigue.

Neural mechanisms associated with vibration-induced fatigue

Cutaneous, muscle spindle and joint receptors are sensitive to vibration exposure. The sensory receptors encode the mechanical stimulation and send these messages along exteroceptive and proprioceptive neural pathways to the motoneurons via peripheral loops and to the central nervous system via supraspinal loops.

Cutaneous pathways have a strong facilitatory influence on motoneurons of flexor muscles. In addition, the primary and secondary endings of the muscle spindles, connected to Ia and II afferent fibers respectively, are very sensitive to the vibratory stimulus (Burke et al. 1976; Roll et al. 1989) and their strong facilitatory influence activates the motoneurons. This vibration-induced contraction, mediated primarily by the Ia monosynaptic and polysynaptic pathways (Desmedt and Godaux 1978; Romaiguère et al. 1991) and cutaneous pathways (Abbruzzese et al. 1978; Romaiguère et al. 1991), is known as the tonic vibration reflex. The secondary endings and joint afferents, in addition to facilitating input to the motoneurons, send messages to the gamma motoneurons. These messages increase the sensitivity of the spindles, which increases the gain of the gamma loop (Johansson et al. 1989; Johansson and Sojka 1991) and thus further increases facilitatory input to the alpha motoneuron. All of these "positive feedback" systems act to facilitate motoneurons' accessibility and increase contraction in the flexor muscles. Simultaneously, an increase in antagonist muscle co-contraction occurs in order to maintain the required grip force output, as observed in earlier studies (Radwin et al. 1987; Park and Martin, 1993). Hence, this reorganization of the motor command of peripheral and central origins increases the overall muscle tension and contributes to the development of fatigue.

\section{Forced firing}

Based on the Cinderella hypothesis proposed by Hägg (1991) and supported by other studies (Sjøgaard et al. 1996), muscle activity in manual work solicits the continuous activity of the same pool of muscle fibers. The process of muscle fiber recruitment and de-recruitment is based on fiber type and its susceptibility to fatigue (Henneman and Mendell 1981). It is hypothesized that the vibration-induced facilitatory drive, as described above, forces the continuous firing of some motor units, which eventually leads to an inability of the muscle fibers to maintain the contraction, resulting in fatigue. This hypothesis is supported by the synchronization of motor units firing under vibration stimulation (Lebedev and Polyakov 1992; Park and Martin 1993) that persists for over 15 min during vibration (Park and Martin 1993). This demonstrates the powerful and long-lasting drive of the vibratory stimulus. Furthermore, the additional recruitment of muscle fibers also contributes to an increase in muscle tension, yet their inherent susceptibility to fatigue means they cannot sustain increases in muscle loads imposed on the system and thus themselves contribute to fatigue.

\section{Biomechanical effects}

All of the aforementioned responses to vibration exposure combine to increase muscle tension. In addition, a biomechanical effect of the $7 \mathrm{~mm}$ vibration displacement amplitude can be observed when the subjects stabilize the forearm while gripping the handle of the vibrator. The forearm muscle mass displacement occurs and the subjects must stabilize this posture, which requires an increase in muscle co-contraction, and simultaneously maintain a $5 \%$ MVC grip on the handle. Hence, the hand-arm system stiffens, enabling increased transmissibility of the vibration through the system., More effort is then required to maintain the grasp on the handle, contributing to increased fatigue. An increase in transmissibility is likely to increase the stretching of the muscle spindles, which constitutes the facilitatory feedback mentioned above.

\section{Intermittent versus sustained grip exertion}

There was an influence based on the type of grip exertion. In the IV condition fatigue was observed 30 and 60 min post-work, whereas in the INV condition fatigue was negligible. These two conditions also show that recovery to pre-work conditions occurs more rapidly than in the sustained grip exertion conditions, with and without the influence of vibration. Therefore, the benefit of complete rest in the work cycles was substantiated as less fatigue and more complete recovery was observed. In addition, rest significantly minimizes the influence of vibration. 
Perception of muscle fatigue

As previously stated, the LFF method was adapted to measure long-lasting fatigue phenomena resulting from low-force static and dynamic work with the hands (Johnson et al. 1995). The subjective ratings indicated that the perception of muscle fatigue was only perceived during and immediately following the work task for all conditions, yet LFF measures showed that the greatest reduction in twitch force magnitude occurred 30-60 min post-work. This suggests that the subjects' perception of fatigue may be more strongly coupled to high frequency fatigue, which is known to recover more rapidly in this context (Moussavi et al. 1992) and that they did not perceive the long-lasting effects of LFF. Therefore, it appears that LFF may not be associated with a specific perception.

\section{Conclusion}

Work task components such as vibration exposure in combination with low grip force exertions may result in the persistence of low-frequency muscle fatigue. The results demonstrated that this type of fatigue was longlasting and was not detected by the subject. The absence of awareness of the persistence of this type of fatigue, and its eventual accumulation, may contribute to an alteration of soft tissue structures and increase the risk for cumulative MSDs.

It is suggested that the use of vibrating tools be specifically evaluated in terms of physical exposure levels, such as vibration frequency and magnitude, grip force exertion, and work cycles to determine the potential influence and interactions of these parameters on muscle fatigue and fatigue accumulation. It seems that some component of rest into the work cycle would be beneficial in reducing the risk associated with certain work tasks, particularly those with a vibratory component. The specific influence of vibration has been shown to increase overall muscle tension and subsequent increase in fatigue and recovery time.

Acknowledgements Support for this research was provided by the National Institute on Disability and Rehabilitation Research of the United States Department of Education, Grant no. H133E980007, "Rehabilitation Engineering Research Center" at the University of Michigan, and research funding by the Occupational Therapy Department at Wayne State University. The opinions contained in this publication are those of the grantee and do not necessarily reflect those of the United States Department of Education.

\section{References}

Abbruzzese G, Hagbarth KE, Homma I, Wallin U (1978) Excitation from skin receptors contributing to the tonic vibration reflex in man. Brain Res 150:194-197

Armstrong TJ, Marshall MM, Martin BJ, Foulke JA, Grieshaber DC, Malone G (2002) Exposure to forceful exertions and vibration in a foundry. Int $\mathbf{J}$ Ind Ergon (in press)
Bigland-Ritchie B, Jones D, Wood J (1979) Excitation frequency and muscle fatigue: electrical responses during human voluntary and simulated contraction. Exp Neurol 64:414-427

Bongiovanni LG, Hagbarth KE (1990) Tonic vibration reflexes elicited during fatigue from maximal voluntary contractions in man. J Physiol (Lond) 423:1-14

Bovenzi M, Fiorito A, Volpe C (1987) Bone and joint disorders in the upper extremities of chipping and grinding operators. Int Arch Occup Health 59:189-198

Burke D, Hagbarth KE, Lofstedt L, Wallin BG (1976) The responses of human muscle spindle endings to vibration of noncontracting muscles. J Physiol (Lond) 261:673-693

Bystrom S, Kilbom A (1991) Electrical stimulation of human forearm extensor muscles as an indicator of handgrip fatigue and recovery. Eur J Appl Physiol 62:363-368

Desmedt JE, Godaux E (1978) Mechanism of the vibration paradox: excitatory and inhibitory effects of tendon vibration on single muscle motor units in man. J Physiol (Lond) 285:197-207

Edwards RHT, Hill DH, Jones DA, Merton PA (1977) Fatigue of long duration in human skeletal muscle after exercise. J Physiol (Lond) 272:769-778

Engstrom K, Dandanell R, Hammerby S, Lindberg J (1990) Bone changes of the hands and exposure conditions among riveters in the aircraft industry. In: Okada A, Taylor W, Dupuis H (eds) Hand-arm vibration. Kanazawa, Japan, pp 31-33

Enoka R, Stuart DG (1992) Neurobiology of muscle fatigue. J Appl Physiol 72:1631-1648

Gardner SH, Hicks AL, McComas AJ (1989) Prolongation of twitch potentiating mechanism throughout muscle fatigue and recovery. Exp Neurol 103:277-281

Gauthier GM, Roll JP, Martin BJ, Harley F (1981) Effects of whole body vibration on sensory motor system performance in man. Aviat Space Environ Med 52:473-479

Gerard M, Martin BJ (1999) Post-effects of long-term vibration on visuo-manual performance in a tracking task. Ergonomics 42:314-326

Goodwin GM, McCloskey DI, Matthews PBC (1972) The contribution of muscle afferents in kinesthesia shown by vibrationinduced illusions of movement and by the effects of paralysing joint afferents. Brain 95:705-748

Green HJ, Jones SR (1989) Does post-tetanic potentiation compensate for low frequency fatigue? Clin Physiol 9:499-514

Hagbarth KE, Eklund G (1966) Tonic vibration reflexes (TVR) in spasticity. Brain Res 2:201-203

Hagberg M, Silverstein B, Wells R, Smith M, Hendrick H, Carayon P, Perusse M (1995) In: Kuorinka I, Forcier L (eds) Work related musculoskeletal disorders (WMSDs): a reference book for prevention. Taylor and Francis, London

Hägg GM (1991) Static workloads and occupational myalgia - a new explanation model. In: Anderson PA, Hobart OJ, Danoff JV (eds) Electromyographical kinesiology. Elsevier, Amsterdam, pp 141-144

Henneman E, Mendell LM (1981) Functional organization of the motoneurone pool and its inputs. In: Brookhart GM, Mountcastle VB (eds) Brooks VB (vol ed) Handbook of physiology. The nervous system: motor control. Oxford University Press, Oxford, pp 423-508

Johansson H, Sojka P (1991) Pathophysiological mechanisms involved in genesis and spread of muscular tension in occupational muscle pain and in chronic musculoskeletal pain syndromes: a hypothesis. Med Hypotheses 35:196-203

Johansson H, Sjolander P, Sojka P, Wadell I (1989) Reflex actions on the muscle spindle systems of muscles acting at the knee joint elicited by stretch of the posterior cruciate ligament. NeuroOrthopaedics 8:9-21

Johansson RS (1978) Tactile sensitivity in the human hand: receptive field characteristics of mechanoreceptor units in the glabrous skin. J Physiol (Lond) 281:101-123

Johnson PW (1998) The development, characterization and implementation of a technique to measure muscle fatigue during computer use. Doctoral dissertation, University of Berkeley, San Francisco 
Johnson PW, Lehman SL, Rempel DM (1995) A new technique for measuring fatigue in the finger flexor muscles resulting from low force work. Proceedings of Premus 2nd International Congress on Prevention of Work-related Musculoskeletal Disorders, Montreal, vol 3, pp 298-300

Jones LA, Hunter IW (1985) Effect of muscle-tendon vibration on the perception of force. Exp Neurol 87:35-45

Lebedev MA, Polyakov AV (1992) Analysis of surface EMG of human soleus muscle subjected to vibration. J Electromyogr Kinesiol 2:1-10

Martin BJ, Armstrong TJ (1995) Contribution of vibration- induced alterations of neurosensory mechanisms to tissue stress and muscle fatigue: a conceptual model. In: Gemne, Bramme, Hagberg, Lundstrom, Nilsson (eds) Hand-arm vibration syndrome. Diagnostics and quantitative relationships to exposure. Arbete och Halsa, pp 97-107

Martin BJ, Park HS (1997) Analysis of the tonic vibration reflex: influence of vibration variables on motor unit synchronization and fatigue. Eur J Appl Physiol 75:504-511

Martin BJ, Gauthier GM, Roll JP, Hugon M, Harlay F (1980) Effects of whole body vibration on standing posture in man. Aviat Space Environ Med 52:778-787

Martin BJ, Roll JP, Di Renzo N (1991) The interaction of hand vibration with oculo-manual coordination in pursuit tracking. Aviat Space Environ Med 62:145-152

Mellor R, Stokes MJ (1992) Detection and severity of low frequency fatigue in the human adductor pollicis muscle. J Neurol Sci 108:196-201

Moussavi RS, Miller RG, Lehman SL (1992) Dantrolene sodium produces fatigue of long duration. Muscle Nerve 15:385-389
Park H, Martin BJ (1993) Contribution of the tonic vibration reflex to muscle stress and muscle fatigue. Scand J Work Environ Health 19:35-42

Pelmear P, Taylor W, Wasserman DE (eds) (1982) Hand-arm vibration: a comprehensive guide for occupational health professionals. Van Nostrand Reinhold, New York

Radwin RG, Armstrong TJ, Chaffin DB (1987) Power hand tool vibration effects on grip exertions. Ergonomics 30:833-855

Roll JP, Vedel JP, Gilhodes JC, Ribot E (1986) Proprioceptive sensory coding of motor activities in man. In: Jeannerod M, Ron S, Schmidt R (eds) Sensorimotor plasticity: theoretical, experimental and clinical aspects. INSERM, Paris, pp 283-304

Roll JP, Vedel JP, Ribot E (1989) Alteration of proprioceptive messages induced by tendon vibration in man: a microneurographic study. Exp Brain Res 76:213-222

Romaiguère P, Vedel JP, Azulay JP, Pagni S (1991) Differential activation of motor units in the wrist extensor muscles during the tonic vibration reflex in man. J Physiol (Lond) 444:645-667

Sandercock TC, Faulkner JA, Albers JW, Abbrect PH (1985) Single motor unit and fiber action potentials during fatigue. J Appl Physiol 58:1073-1079

Sjøgaard K, Christensen H, Jensen BR, Finsen L, Sjøgaard G (1996) Motor control and kinetics during low level concentric and eccentric contractions in man. Electroencephalogr Clin Neurophysiol 101:453-460

Vandervoort AA, Quinlan J, McComas AJ (1983) Twitch potentiation after voluntary contraction. Exp Neurol 81:141-152

Vøllestad NK, Sejersted OM (1988) Biomedical correlates of fatigue. Eur J Appl Physiol 57:336-347 